\title{
COVID-19 Data Based on Wavelet Coherence Estimates for Selected Countries in the Eastern Mediterranean
}

\author{
Asaad Ma. Babker ${ }^{1}$, Vyacheslav Lyashenko ${ }^{2}$ \\ 1 Department of Medical Laboratory Sciences, College of Health Sciences, Gulf Medical University, Ajman, UAE \\ 2 Department of Informatics, Kharkiv National University of Radio Electronics, Ukraine \\ *Corresponding Author: Dr. Asaad Ma. Babker, Department of Medical Laboratory Sciences, College of Health Sciences, Gulf \\ Medical University, Ajman, UAE \\ Email: azad.88@hotmail.com; Tel: 00971527900035
}

Received: April 30, 2020; Accepted: May 11, 2020

\section{Abstract}

Background and Objective: The development of the COVID-19 pandemic makes it necessary to conduct various studies on this topic. One of the key questions is the study of the dynamics of the development of this disease. It is important to know for each country. At the same time, the study of the dynamics of the development of COVID-19 for countries in a particular region is relevant. The main objective of this study is to analyze the main indicators of the development of the COVID-19 epidemic for individual countries in the eastern Mediterranean.abbreviations if possible. Materials and Methods: We review statistics that characterize the total number of confirmed cases of COVID-19, total number of recovered, total number of deaths. This is cumulative data. These data are considered for each individual country from the selected region. Also summarized data for the selected region are considered. To analyze the data, we use estimates of the wavelet coherence values. Results: We obtained estimates of wavelet coherence values for countries such as: Egypt, Israel, Jordan, Lebanon, Syria, Turkey and Cyprus. These estimates reflect the depth of the relationship between total number of confirmed cases of COVID-19 and total number of recovered, between total number of confirmed cases of COVID-19 and total number of deaths. This makes it possible to assess the degree of influence between the series of data that are being investigated. This allows us to draw conclusions about the development of the COVID-19 pandemic. Conclusions: The results are obtained that explain some aspects of the dynamics of the COVID-19 pandemic in individual countries of the selected region.

Keywords: Viruses, COVID-19, Pandemic, Wavelet analysis, Wavelet coherence, Data series.

\section{INTRODUCTION}

Various diseases accompany a person throughout his life. Thus, diseases are an integral part of human life. Of particular importance, in this context, are epidemics and pandemics, which are capable of rapidly spreading and affecting wide sections of the population1. It is also important to note that in the early stages of the development of epidemics and pandemics, there are no drugs to combat them.

In its history, humanity has experienced many epidemics and pandemics1. Currently, humanity is faced with coronavirus infection COVID-19. COVID-19 is a severe acute respiratory infection caused by the SARS-CoV-2 virus2. As of 21.04.2020, 2397217 people were infected with COVID-19, 162956 people died3. However, less than 4 months have passed since the pandemic began.
One of the areas of study of epidemics and pandemics is the study of the dynamics of the spread of the disease, the dynamics of deaths and the dynamics of recovered patients. Such indicators are the main ones in the study of the development of the epidemic (pandemics) [4]. At the same time, this analysis allows you to:

- make assumptions about possible peaks in the development of the epidemic (pandemic);

- to predict the dynamics of the epidemic (pandemic);

- to analyze the consistency of individual disease indicators, which allows us to draw general conclusions about the effectiveness of measures that are used to stop the development of the epidemic and treatment methods. 
Thus, the main goal of this study is to analyze the main indicators of the development of the COVID-19 epidemic for individual countries. An important point of this analysis is also a comparison of the development of the COVID-19 epidemic between different countries.

\section{MATERIALS AND METHODS}

A small review of research publications: To study the dynamics of the epidemic, as a rule, various statistical methods are used.

Wang et al.5 to study the dynamics of COVID-19, a predictive model is used. This model takes into account the rate of transmission of the disease, the frequency of infection, and the rate of recovery. This model allows us to assess the development trends of the COVID-19 epidemic, taking into account preventive measures and counteraction to infection from COVID-19.

Sarkodie and Owusu6 use dynamic statistical analysis techniques to study the dynamics of the epidemic COVID-19. The authors of the study also emphasize the importance of considering data that are derived from descriptive statistics. This allowed them to build various models for the analysis of COVID19 dynamics. Comparison of different models among themselves allows us to describe various cases of the development of COVID-19 dynamics. This allows you to better understand the dynamics of the spread of the epidemic.

Yang et al. [7] use a simple linear regression model to estimate deaths coefficients from COVID-19. Moreover, all cases of deaths were divided into separate groups, depending on the development of the disease. This approach provides simple and quick estimates, which helps to understand the dynamics of COVID-19, to evaluate the effectiveness of measures taken to prevent this disease.

Dehkordi et al. [8] conduct a study of the dynamics of the development of COVID-19 based on classical statistical methods. Such an analysis is carried out for different countries. This allows us to find a common approach for revealing the relationship between the dynamics of diseases and the deaths from COVID-19.

Nishiura, Linton, and Akhmetzhanov [9] examine the validity of empirical data, which is important in the study of COVID-19 dynamics. Statistical methods compare complete data sets and individual subsets of the source data. This comparison is based on various distribution models that describe empirical data. This allows you to minimize errors that may occur in the process of building a forecast for the development of the epidemic.

Brandenburg10 considers various distribution laws to explain the mortality processes during the development of the COVID19 pandemic. The author provides a detailed explanation for the exponential and quadratic growth model of the number of deaths from COVID-19. This allows you to better understand the situation that arises in the process of obtaining new statistics.

Kamrujjaman, Mahmud, and Islam [11] explore the general picture of the development of COVID-19 as part of mathematical growth functions. This makes it possible to understand which growth pattern corresponds to the scenario for individual cases.

Thus, various directions in the study of the development of the COVID-19 epidemic can be distinguished. This allows us to understand such dynamics as a whole. In this case, special attention should be paid to comparative analysis, which will help to better reveal the dynamics of the COVID-19 epidemic. At the same time, such a comparison should be carried out in the context of key indicators, such as: the dynamics of the spread of the disease, the dynamics of deaths and the dynamics of recovered patients.

In our opinion, this can be done based on the methodology of wavelet analysis and the corresponding methods and approaches12-14. Moreover, the wavelet analysis methodology can give new results in the study of the dynamics of the distribution of COVID-19.

Wavelet coherence as one of the tools of the general wavelet methodology: In general, wavelet analysis allows the analysis of various processes where the data are presented in the form of a time series 15. Such an analysis is based on the search for changes in the values of the series being investigated [12]. Among the methods of wavelet ideology, wavelet coherence should be distinguished $[15,16]$. Wavelet coherence allows cross-sectional analysis for the data that is being investigated. This is important for studying the dependencies between different data and determining the depth of such dependence. At the same time, the depth of the relationship between the data has a certain meaning (this will be shown below).

To determine the values of wavelet coherence, we consider the values of cross wavelet spectra $U_{x y}(k, l)$ for two data series $k(t)$ and $l(t)$ ( $x$ is the variable that displays the data number in the series under investigation, $y$ is the variable that displays the depth of cross-links for a time series, $t$ - time interval being investigated). The general formula has the following form 15, 16:

$$
R^{2}(k, l)=\frac{\left|H\left(l^{-1} U_{x y}(k, l)\right)\right|}{H\left(l^{-1}\left|U_{x}(k, l)\right|^{2}\right) H\left(l^{-1}\left|U_{y}(k, l)\right|^{2}\right)},
$$

where: $H$ is a smoothing operator, $0 \leq R^{2}(k, l) \leq 1$. If the values $R^{2}(k, l)$ tend to zero, then we have a weak relationship between data series. Otherwise, we have a strong dependence $[15,16]$.

Data for analysis: For analysis, selected countries of the eastern Mediterranean. Among these countries we consider: Egypt, Israel, Jordan, Lebanon, Syria, Turkey and Cyprus. This region and these countries are chosen because they are one of the destinations for tourism and recreation. Currently, these are countries such as Egypt, Israel, Jordan, Turkey and Cyprus. Other countries are added for comparison and a more complete description of the region. Therefore, it is important to know the dynamics of COVID-19 in this region. This is important from the point of view of security and the possibility of resuming tourism in this area.

The general data for the countries we are considering is publicly available on the websites:

https://www.worldometers.info/coronavirus/, 
https://www.who.int/emergencies/diseases/novel-coronavirus2019/situation-reports,

https://github.com/datasets/covid-19,

https://datahub.io/core/covid-19.

We are considering the period 22.01.2020 - 20.04.2020. We will consider such data: total number of confirmed cases of COVID19 , total number of recovered, total number of deaths. This is cumulative data.

For different countries, data are presented for different periods. This is an objective fact and is associated with differences in the conditions for the onset and spread of the COVID-19 epidemic for each country. Fig. 1 shows the logarithmic dependence of the data.

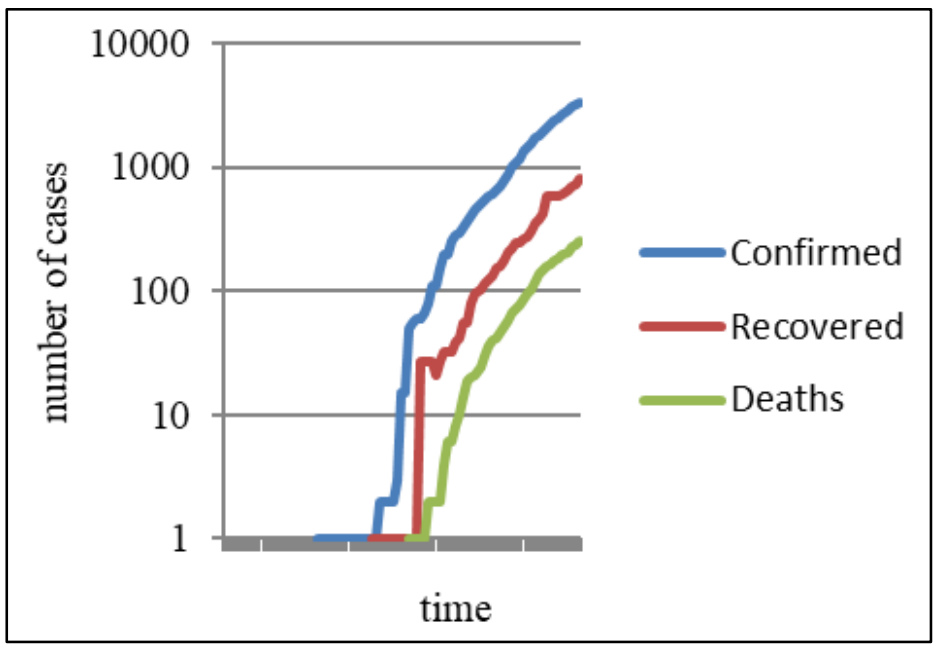

a) Egypt

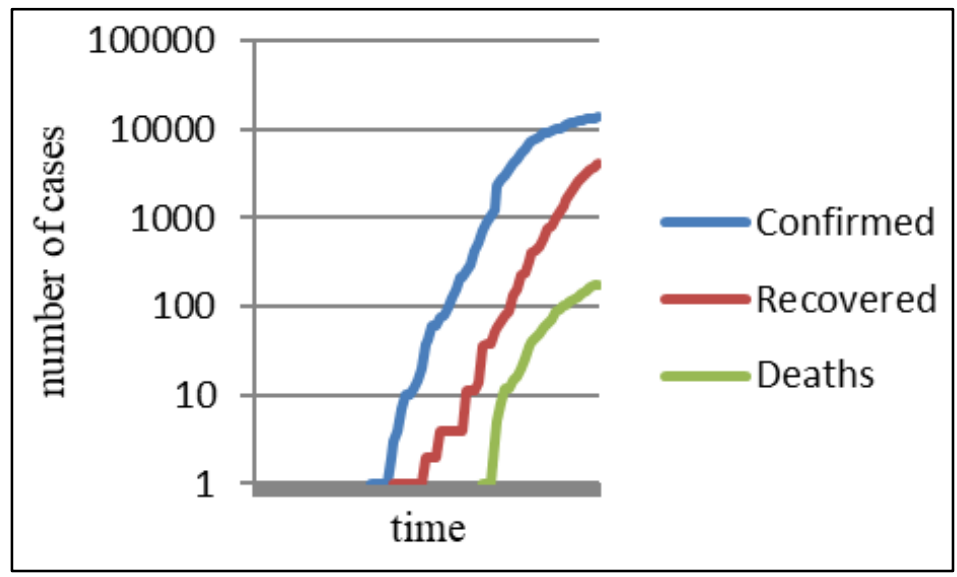

b) Israel

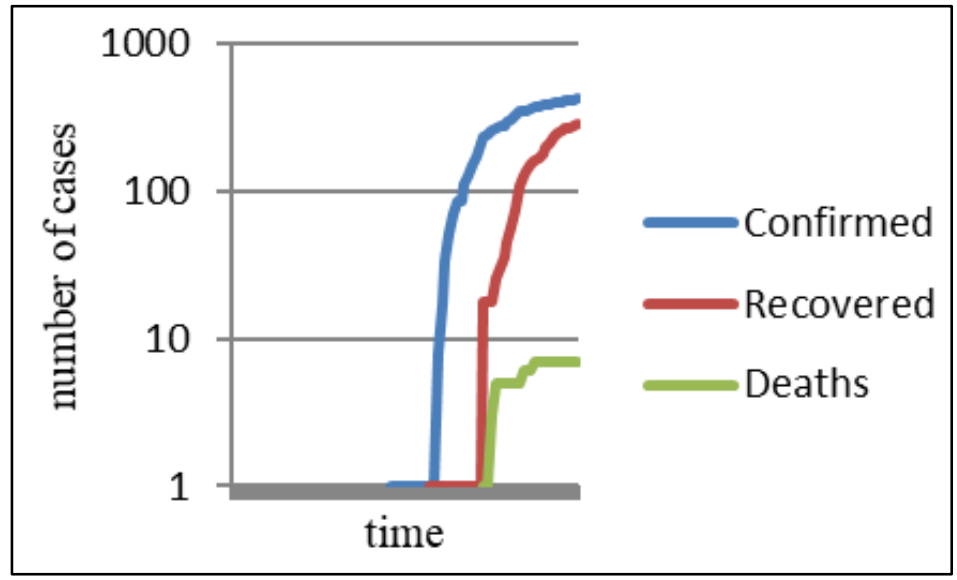

c) Jordan 


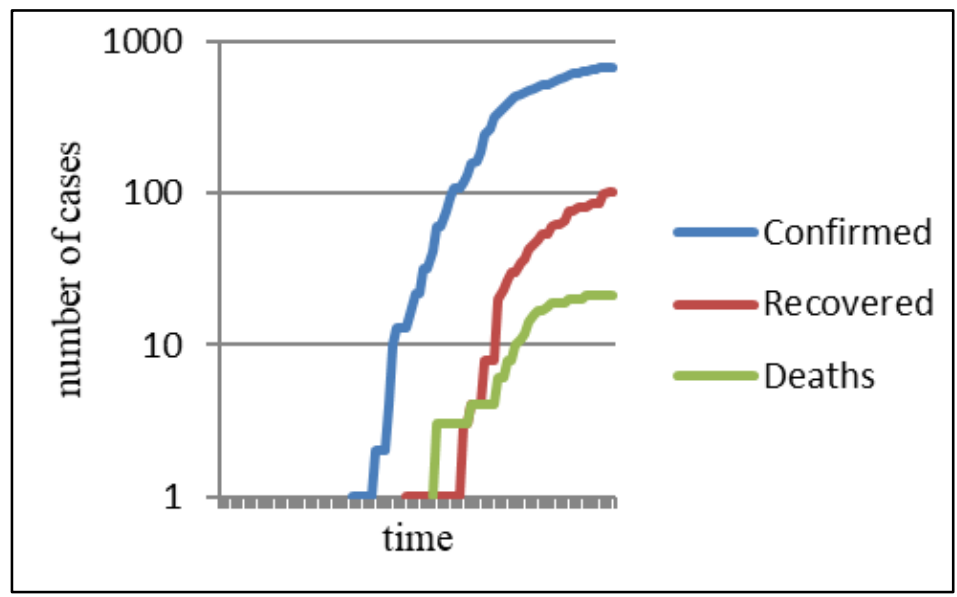

d) Lebanon

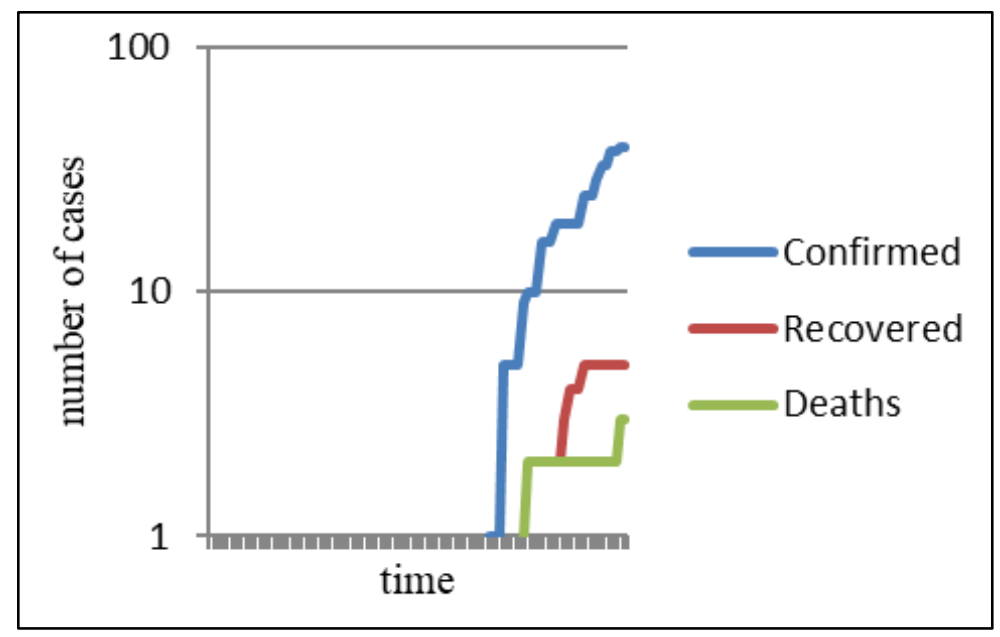

e) Syria

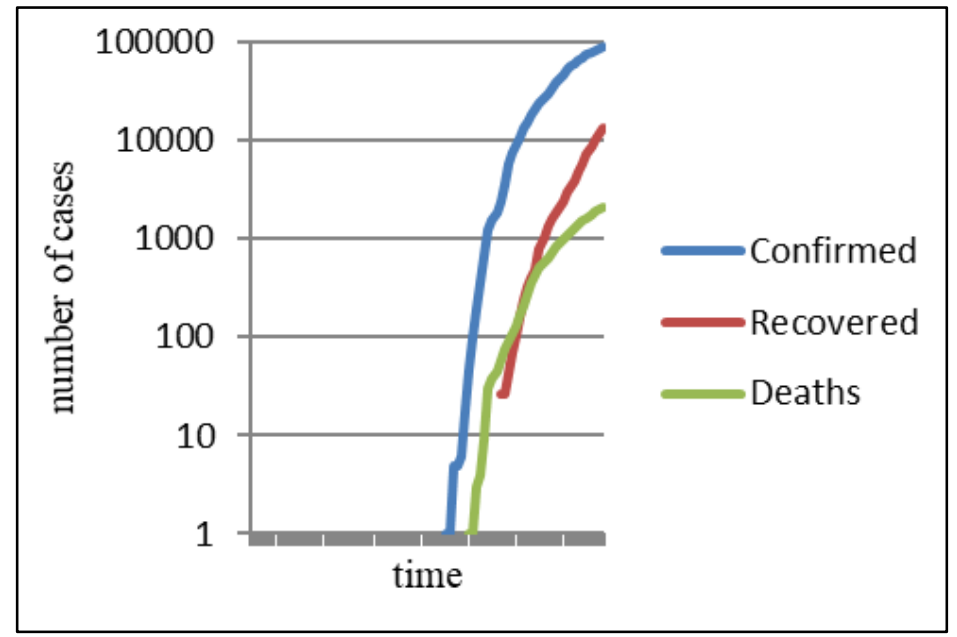

f) Turkey 


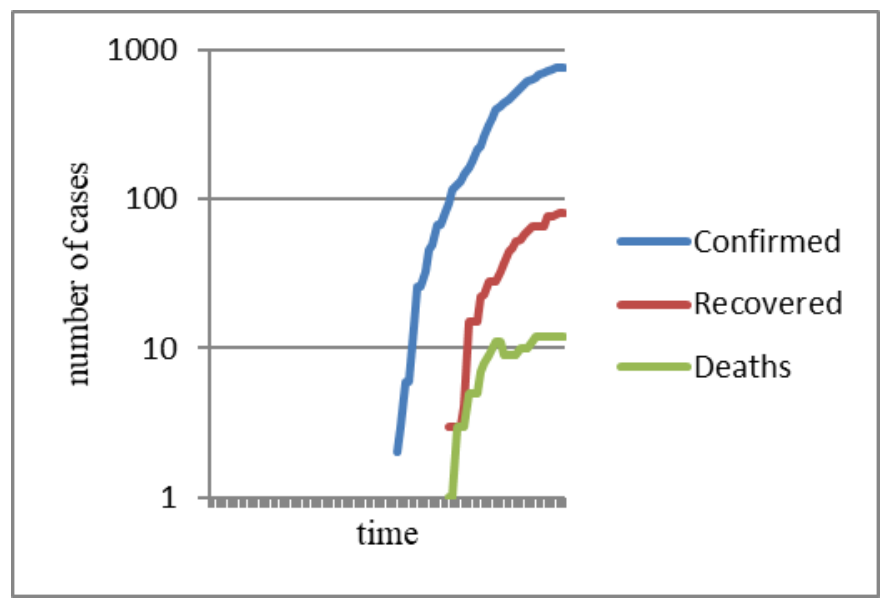

g) Cyprus

Fig 1: Dynamics of the development of the COVID-19 pandemic in selected countries of the eastern Mediterranean (logarithmic dependence)

We can observe the different dynamics of the development of the COVID-19 pandemic. We can also observe completely different dynamics of deaths people from COVID-19 in each country that we analyze. The same differences are observed in the dynamics of recovered patients.

Therefore, for an extended analysis and understanding of the pandemic development processes, we will consider the wavelet coherence estimates between the main indicators that characterize the dynamics of COVID-19. Such estimates are given for each country separately.

\section{RESULTS}

We will consider wavelet coherence estimates between total number of confirmed cases of COVID-19 and total number of recovered, as well as between total number of confirmed cases of COVID-19 and total number of deaths. This will determine the depth of the relationship between each pair of data series that are being investigated. The depth of this relationship can be interpreted:

- between the total number of confirmed cases of COVID-19 and the total number of recovered as a steady number of days necessary for patients to recover,

- between the total number of confirmed cases of COVID-19 and the total number of deaths as the number of days characterizing the possible likelihood of a fatal event.

As a result, this data can be estimates that characterize the onset of outcomes (recovery or deaths) after infection with the COVID-19 coronavirus. At the same time, the depth of the relationship may characterize:

treatment effectiveness (for example, depth-stable periods of the relationship between the total number of confirmed cases of COVID-19 and the total number of recovered for the entire time interval that is analyzed),

the effectiveness of the response of the medical system in countering the spread of COVID-19 (for example, the presence of periods that reflect significant in-depth relationships between the total number of confirmed cases of COVID-19 and total number of deaths),

the presence in the analyzed data of late treatment with signs of COVID-19 disease (in the case of the presence of periods that reflect insignificant in-depth relationships between total number of confirmed cases of COVID-19 and total number of deaths).

It should also be noted that the source data for each country have different lengths. The defining data series is a data series that shows the total number of confirmed cases of COVID-19. Therefore, all other data series will be compared with a data series that shows the total number of confirmed cases of COVID-19. Perhaps in some cases we will not have consistency between some series of data. This can happen when the data series are not large enough or their data is not significant. If this happens, we will discuss it separately.

Fig. 2 - Fig. 8 presents the results for estimating the wavelet coherence for each of the countries we are exploring.

In Fig. 2 - Fig. 8, the abscissa axis indicates the time period, which is considered for each country separately. The measurement interval is one day. The ordinate axis shows the scale of the depth of cross-references - the depth of the relationship between the series of data that are being studied. The relationship period is measured in days. The dashed white line limits the region of reliable values of wavelet coherence (with a confidence level of at least 0.95). Each figure (right) also shows a scale for analyzing the significance of wavelet coherence data (from 0 to 1 ). Such data for clarity have color values [15-18].

In Fig. 2 shows the wavelet coherence values for data from Egypt. These data are for the period 14.02.2020-24.04.2020. Fig. 2a shows the relationship between total number of confirmed cases of COVID-19 and total number of recovered. Fig. $2 \mathrm{~b}$ shows the relationship between total number of confirmed cases of COVID-19 and total number of deaths. 


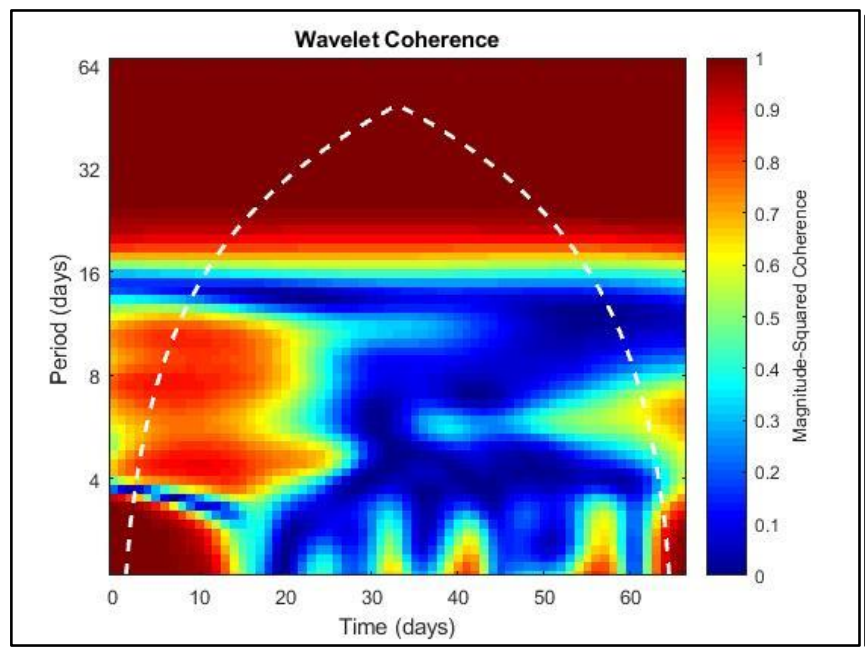

A.

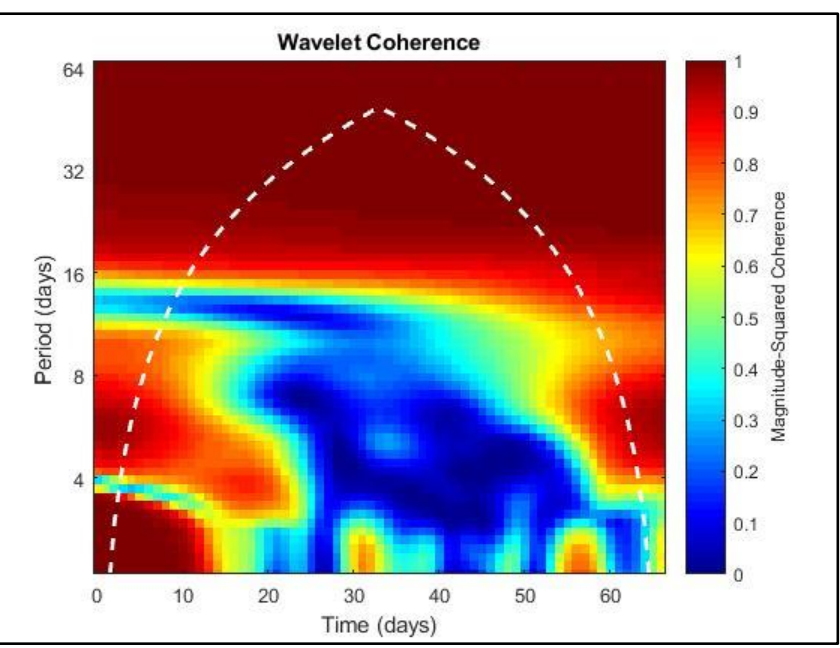

B.

Fig 2: Wavelet coherence values for data from Egypt

In Fig. 3 shows the wavelet coherence values for data from Israel. These data are for the period 21.02.2020-24.04.2020. Fig. $3 \mathrm{a}$ shows the relationship between total number of confirmed cases of COVID-19 and total number of recovered. Fig. $3 \mathrm{~b}$ shows the relationship between total number of confirmed cases of COVID-19 and total number of deaths.

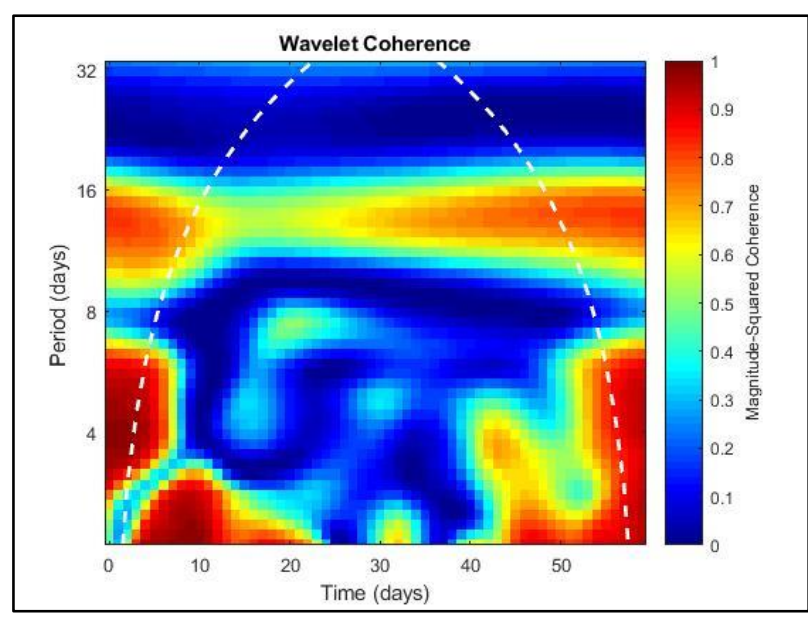

A.

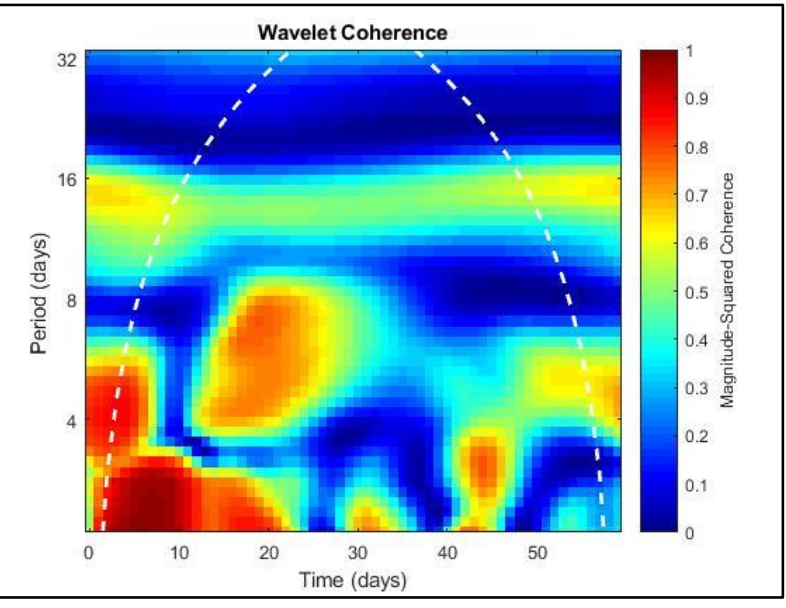

B.

Fig 3: Wavelet coherence values for data from Israel

In Fig. 4 shows the wavelet coherence values for data from Jordan. These data are for the period 03.03.2020-24.04.2020.

Fig. 4a shows the relationship between total number of confirmed cases of COVID-19 and total number of recovered. Fig. $4 \mathrm{~b}$ shows the relationship between total number of confirmed cases of COVID-19 and total number of deaths. 


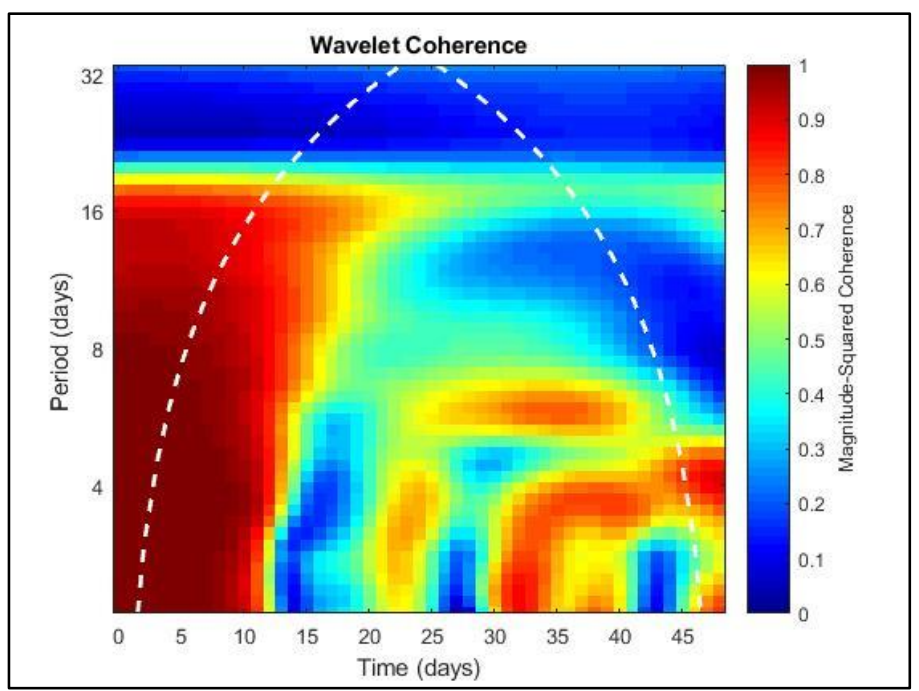

A.

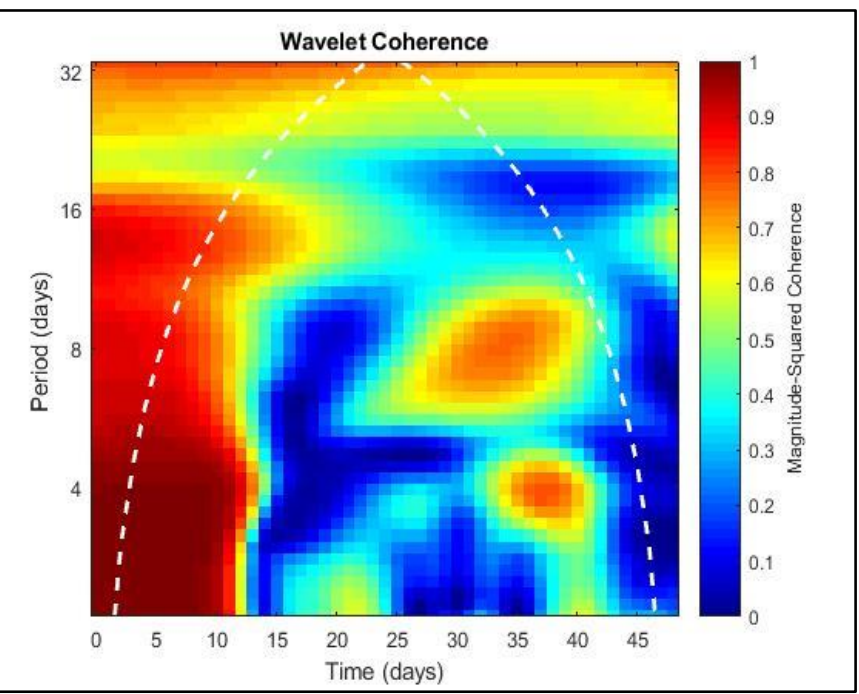

B.

Fig 4: Wavelet coherence values for data from Jordan

In Fig. 5 shows the wavelet coherence values for data from Lebanon. These data are for the period 21.02.2020-24.04.2020. Fig. $5 a$ shows the relationship between total number of confirmed cases of COVID-19 and total number of recovered.
Fig. $5 b$ shows the relationship between total number of confirmed cases of COVID-19 and total number of deaths.

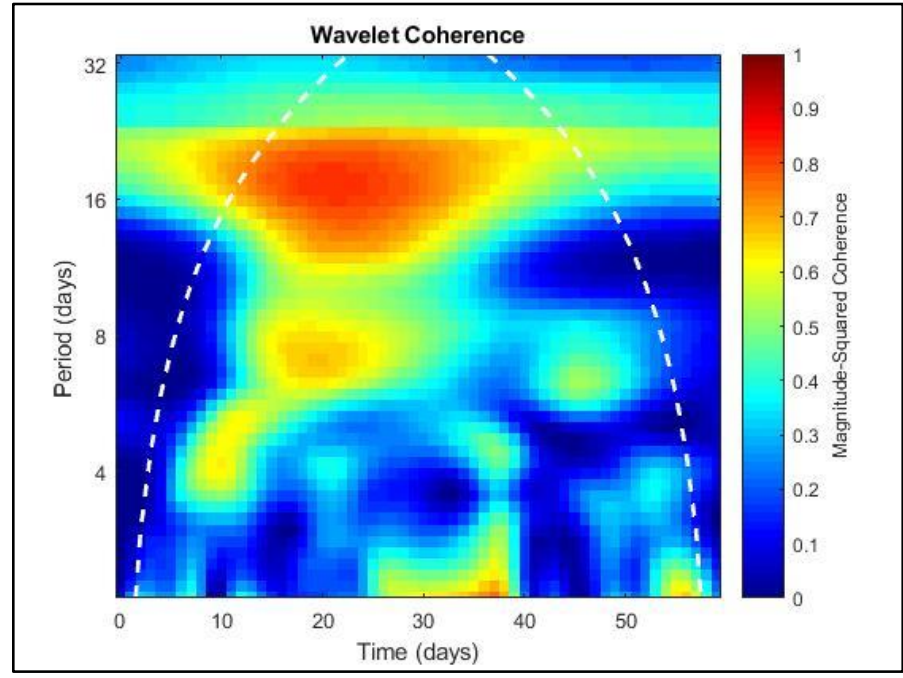

A.

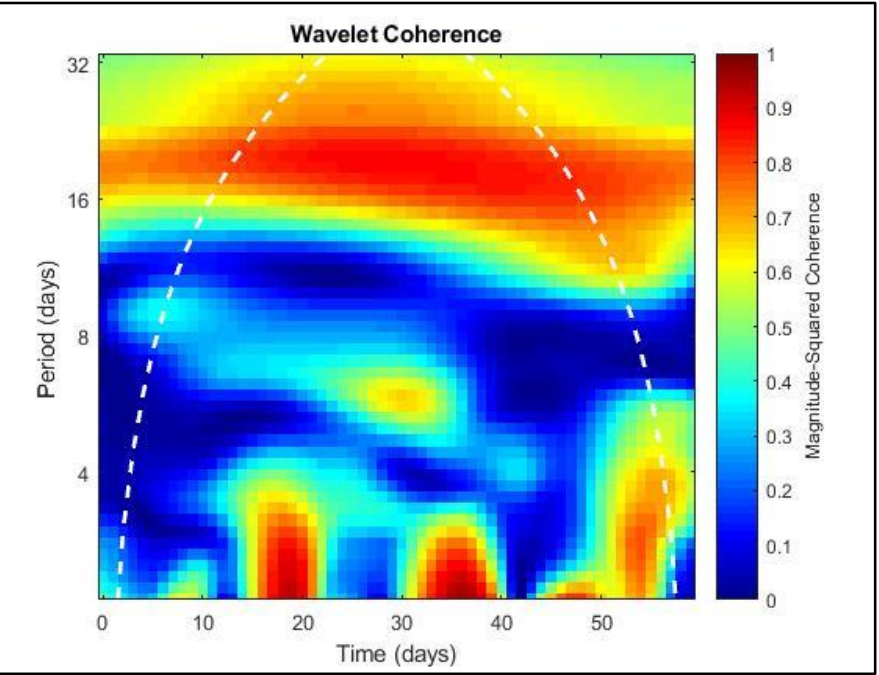

B.

Fig 5: Wavelet coherence values for data from Lebanon

In Fig. 6 shows the wavelet coherence values for data from Syria. These data are for the period 22.03.2020-24.04.2020. Fig. $6 a$ shows the relationship between total number of confirmed cases of COVID-19 and total number of recovered. Fig. 6b shows the relationship between total number of confirmed cases of COVID-19 and total number of deaths. 


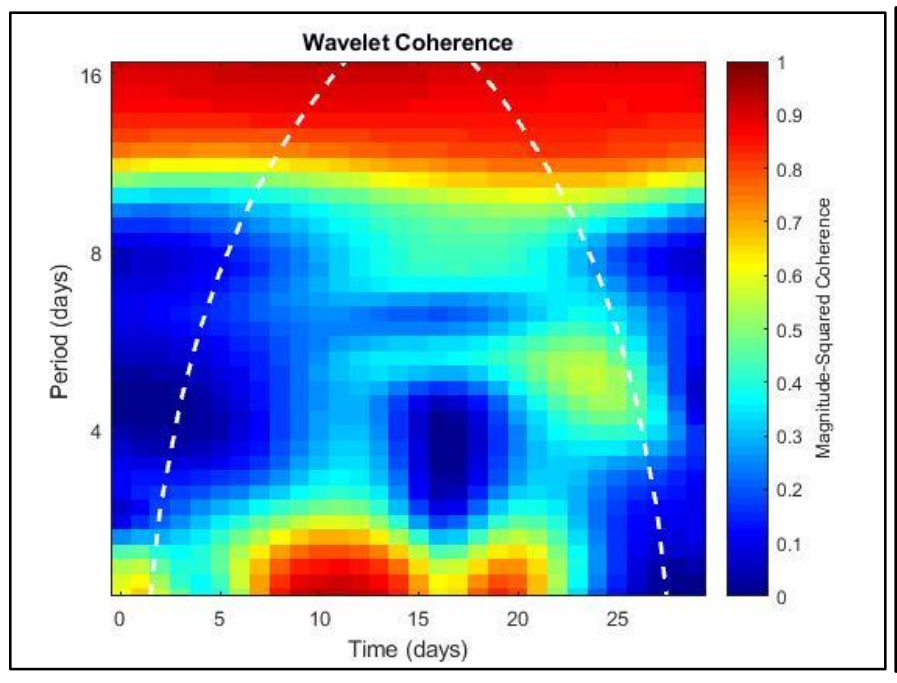

A.

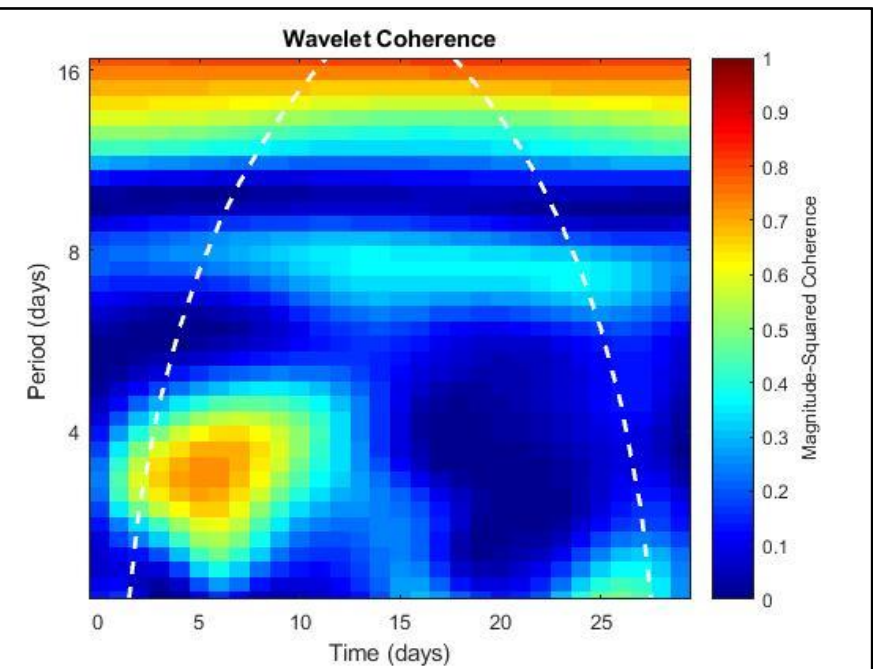

B.

Fig 6: Wavelet coherence values for data from Syria

In Fig. 7 shows the wavelet coherence values for data from Turkey. These data are for the period 11.03.2020-24.04.2020. Fig. $7 \mathrm{a}$ shows the relationship between total number of confirmed cases of COVID-19 and total number of recovered. Fig. 7b shows the relationship between total number of confirmed cases of COVID-19 and total number of deaths.

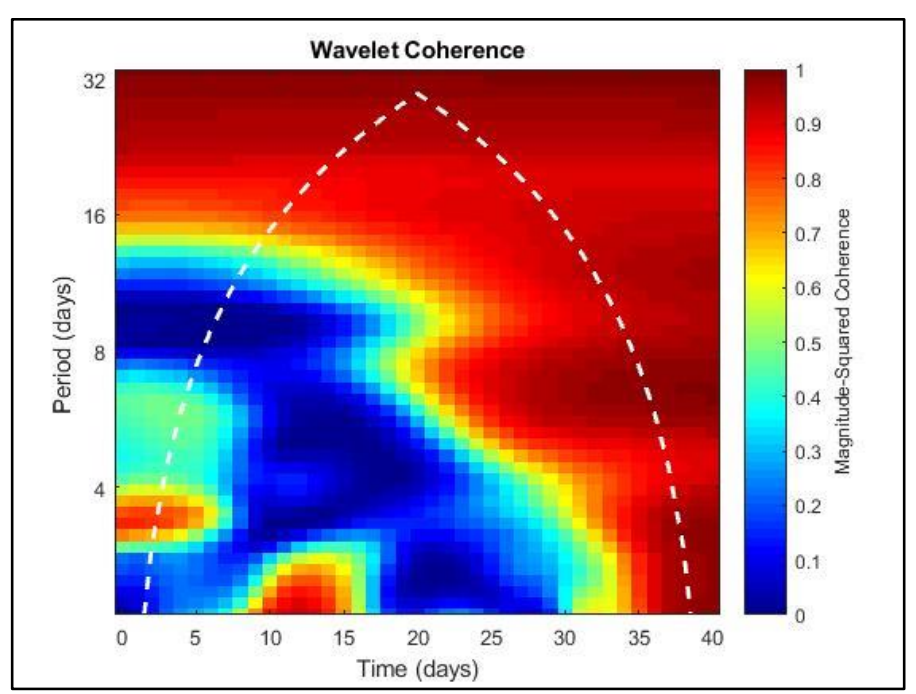

A.

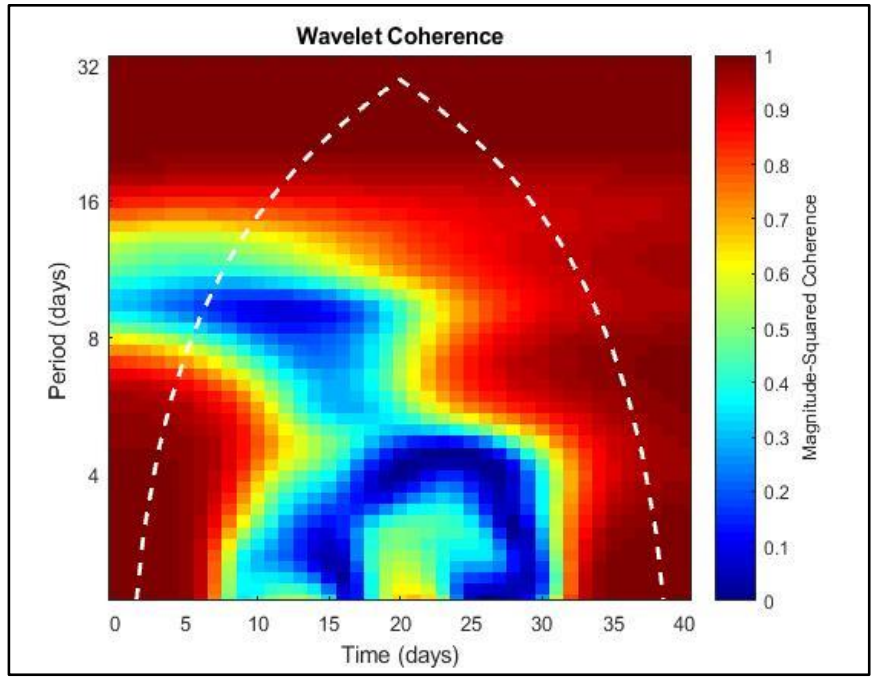

B.

Fig 7: Wavelet coherence values for data from Turkey

In Fig. 8 shows the wavelet coherence values for data from Cyprus. These data are for the period 09.03.2020-24.04.2020. Fig. $8 \mathrm{a}$ shows the relationship between total number of confirmed cases of COVID-19 and total number of recovered. Fig. $8 \mathrm{~b}$ shows the relationship between total number of confirmed cases of COVID-19 and total number of deaths. 


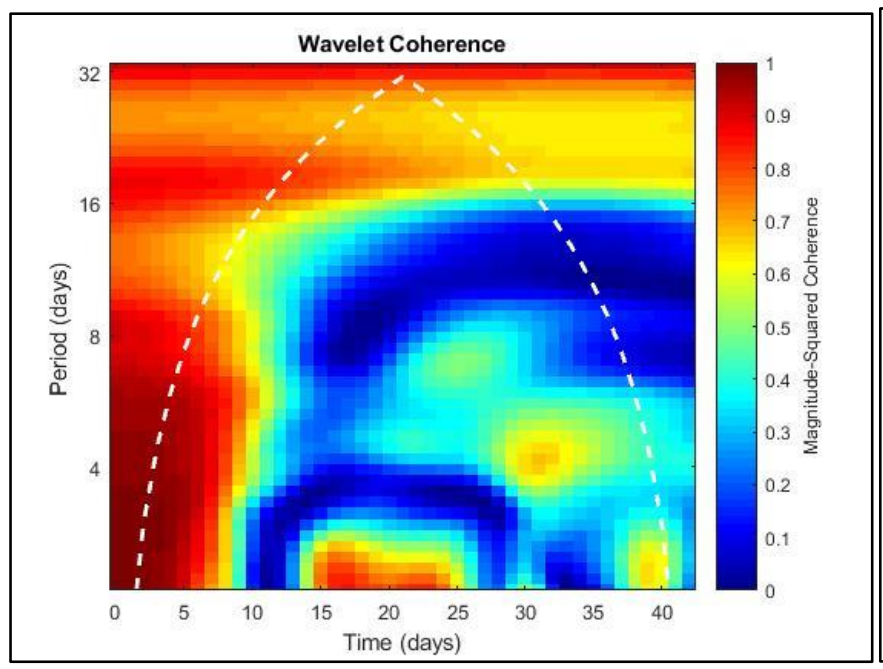

A.

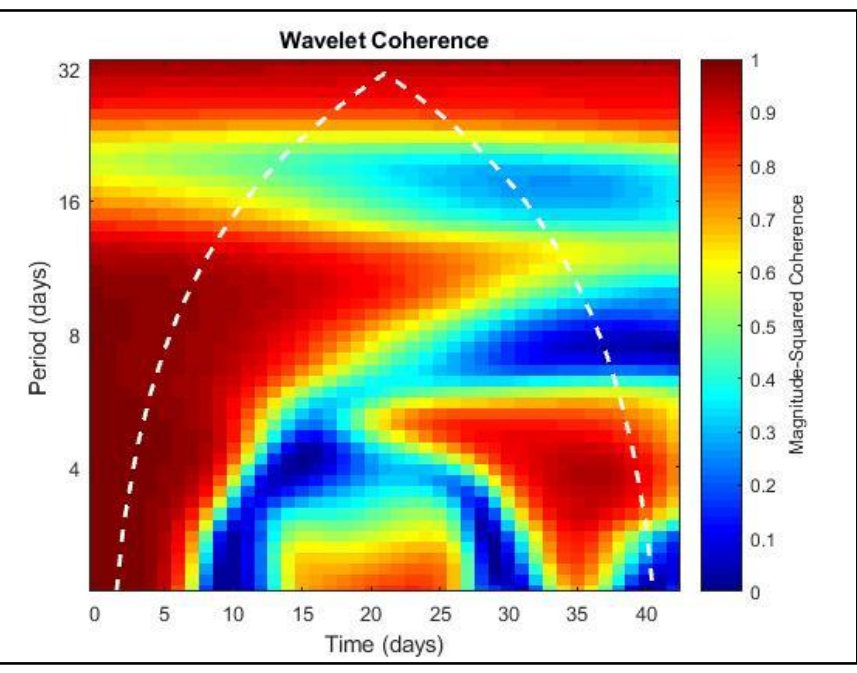

B.

Fig 8: Wavelet coherence values for data from Cyprus

In Fig. 9 shows the generalized wavelet coherence values for the data of all countries in the region that are being studied. These data are for the period 14.02.2020-24.04.2020. Fig. 9a shows the relationship between total number of confirmed cases

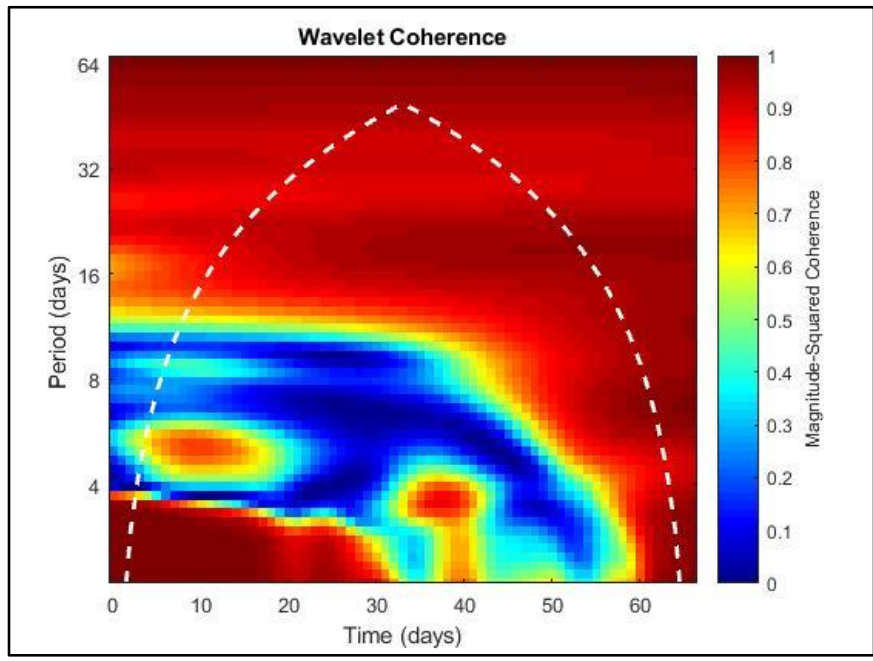

A. of COVID-19 and total number of recovered. Fig. 9b shows the relationship between total number of confirmed cases of COVID19 and total number of deaths.

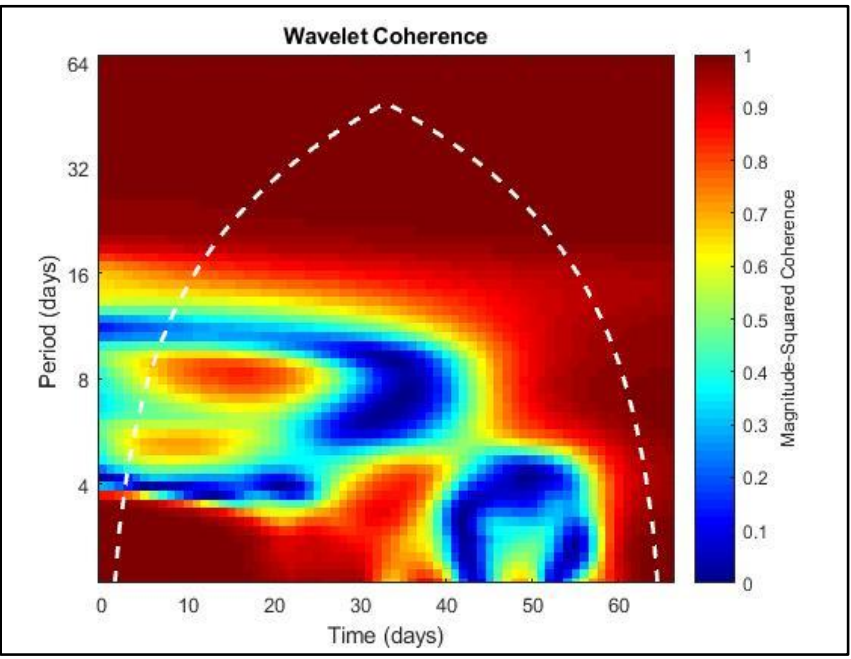

B.

Fig 9: Wavelet coherence values for data of all countries in the region that are being studied

We see that the values of the wavelet coherence estimates are different. But we can also see the same trends for different countries. We discuss these results in more detail.

\section{DISCUSSION}

First of all, we note that not all wavelet coherence estimates have high significant values over the entire time interval that is studied. This is what we assumed. This is especially true for data from Syria (see Fig. 6). Most likely, this is due to the situation in this country and the insufficient data provided. Similarly, we can speak about the values of the wavelet coherence estimates for the data from Lebanon (see Fig. 5).

For other countries, the following can be said.
For data from Egypt, we can say that the depth of the stable relationship between the total number of confirmed cases of COVID-19 and the total number of recovered starts from 16-17 days (see Fig. 2). Therefore, it can be assumed that the expected recovery of patients with COVID-19 will be after this treatment period. At the same time, the depth of the stable relationship between the total number of confirmed cases of COVID-19 and the total number of deaths for data from Egypt has different meanings. This can be interpreted as either a late call for help, or the complexity of the course of the disease COVID-19. However, it can also be said that there are periods when a significant and stable cross-reference depth in terms of the possible death of patients with COVID-19 is 16 days.

Ambiguous results are from Israel (see Fig. 3). Given the level of medicine in Israel, we see that the depth of the relationship 
between total number of confirmed cases of COVID-19 and total number of recovered is fragmented. At the same time, the stability of such a relationship is observed between 12-16 days of the course of the disease. At the same time, the depth of the relationship between the total number of confirmed cases of COVID-19 and the total number of deaths for data from Israel is small (up to 8 days). This can be interpreted as the fact that death from COVID-19 occurs in especially severe cases that are difficult to treat.

For data from Jordan (see Fig. 4), the relationship between total number of confirmed cases of COVID-19 and total number of recovered is most significant at the beginning of the study time period. However, for later periods, this relationship is less significant. The explanation for this may be either a lack of data or the initial stage of the development of the COVID-19 epidemic. Similar findings apply to the relationship between total number of confirmed cases of COVID-19 and total number of deaths for data from Jordan. But here it is also necessary to consider the insignificant total number of deaths. In this case, deaths are characteristic after 4 or 8 days of illness.

For data from Turkey (see Fig. 7), the stability and significance of the relationship between the total number of confirmed cases of COVID-19 and the total number of recovered is characteristic of a later period from the studied time interval. The relationship between total number of confirmed cases of COVID-19 and total number of deaths for data from Turkey is significant and stable at the beginning and end of the study time period. At the beginning of the period, such a relationship occurs within 8 days of detecting COVID-19. At the end of the period, this relationship has a different time frame (from 1 to 32 days). This indicates the complexity of the COVID-19 epidemic in Turkey, the inadequacy of the measures that are being taken.

For data from Cyprus (see Fig. 8), the relationship between total number of confirmed cases of COVID-19 and total number of recovered is most stable after 16 days of the course of the disease. At the same time, the relationship between total number of confirmed cases of COVID-19 and total number of deaths has the greatest stability at 15 days (at the beginning period, which analyzed) and from 3 to 5 days (in the middle and end period, which analyzed). However, a small number of deaths should also be considered. However, these deaths are most likely caused by severe complications or late seeking help.

Fig. 9 gives a general idea of the development of COVID-19 in the region. These data can be interpolated in the absence of data for different countries or to supplement the results. In general, the data in Fig. 9 indicate the complexity of the course of the COVID-19 pandemic in the region (see Fig. 9b).

The data obtained correlate with the results of other researchers. In the work of Ronco, Navalesi and Vincent19 it was shown that a possible recovery (depending on the severity of the disease) by the average number of days from the onset of symptoms is possible after 14 days of hospitalization. At the same time, the average number of days before death can be minimal (depending on the length of hospitalization and the time the infection was detected) $[20,21]$.

\section{CONCLUSION}

We analyzed statistics that describe the dynamics of the development of the COVID-19 pandemic in selected countries in the eastern Mediterranean region. The possibility and expediency of using wavelet methodologies for conducting the corresponding analysis are shown. As such a tool, wavelet coherence estimates are used. This method makes it possible to assess the degree of influence between the data series that are being investigated. The results are obtained that explain some aspects of the dynamics of the COVID-19 pandemic in individual countries of the selected region. The results of wavelet coherence for generalized data are also obtained. These data can be used as boundary criteria.

\section{Conflict of Interest: None.}

\section{REFERENCES}

1. Hays JN, Epidemics and pandemics: their impacts on human history. Santa Barbara, California: ABC-CLIO, ISBN: $1851096639,2005$.

2. Lai CC, Shih TP, Ko WC, Tang HJ, Hsueh PR, Severe acute respiratory syndrome coronavirus 2 (SARS-CoV-2) and corona virus disease-2019 (COVID-19): the epidemic and the challenges. Int. J. Antimicrob. Agents, 2020; 55(3):105924. 10.1016/j.ijantimicag.2020.105924

3. World Health Organization, 2020. Coronavirus disease 2019 (COVID-19): situation report, 92.

4. Siettos $\mathrm{Cl}$, Russo L. Mathematical modeling of infectious disease dynamics. Virulence, 2013;4(4):295-306. 10.4161/viru.24041.

5. Wang $\mathrm{H}$, et al. Phase-adjusted estimation of the number of coronavirus disease 2019 cases in Wuhan, China. Cell discov. 2020;6:1-8. 10.1038/s41421-020-0148-0.

6. Sarkodie SA, Owusu PA. Investigating the cases of novel coronavirus disease (COVID-19) in China using dynamic statistical techniques. Heliyon, 2020;6(4):e03747. 10.1016/j.heliyon.2020.e03747.

7. Yang $S$, et al. Early estimation of the case fatality rate of COVID-19 in mainland China: a data-driven analysis. Annals of Translational Medicine, 2020;8(4):128. 10.21037/atm.2020.02.66

8. Dehkordi AH, Alizadeh M, Derakhshan P, Babazadeh $\mathrm{P}$, Jahandideh A. Understanding Epidemic Data and Statistics: A case study of COVID-19. arXiv preprint arXiv:2003.06933, 2020.

9. Nishiura $\mathrm{H}$, Linton NM, Akhmetzhanov AR. Serial interval of novel coronavirus (COVID-19) infections. Int. J. Infect. Dis. 2020;93:284-286. 10.1016/j.ijid.2020.02.060.

10. Brandenburg A. Quadratic growth during the 2019 novel coronavirus epidemic. arXiv preprint arXiv:2002.03638, 2020.

11. Kamrujjaman M, Mahmud MS, Islam MS. Coronavirus outbreak and the mathematical growth map of COVID19. Annu. Rev. Res. Biol., 2020;35(1):72-78. 10.9734/arrb/2020/v35i130182.

12. Lyashenko VV, Babker AMAA, Kobylin OA. The methodology of wavelet analysis as a tool for cytology preparations image processing. Cukurova Medical Journal, 2016;41(3):453-463. 10.17826/cukmedj.237468.

13. Lyashenko VV, Babker AMAA, Kobylin OA. Using the Methodology of Wavelet Analysis for Processing Images of Cytology Preparations. Natl. J. Med. Res. 2016;6:98-102.

14. Lyashenko VV, Babker MA, A Lyubchenko VA. Wavelet Analysis of Cytological Preparations Image in Different Color Systems. Open Access Library Journal, 2017;4:e3760. 10.4236/oalib.1103760.

15. Torrence C, Webster PJ. Interdecadal changes in the ENSO-monsoon system. J. clim., 1999;12(8):2679-2690. 10.1175/1520-0442(1999)012<2679:ICITEM>2.0.CO;2.

16. Ng EK, Chan JC. Geophysical applications of partial wavelet coherence and multiple wavelet coherence. J. Atmos. Ocean. Tech., 2012;29(12):1845-1853. 
10.1175/JTECH-D-12-00056.1.

17. Ahmad MA, Mustafa SK, Zeleniy O, Lyashenko V. Wavelet Coherence as a Tool for Markers Selection in the Diagnosis of Kidney Disease. Int. J. Emerg. Trends Eng. Res., 2020; 8(2):378-383. 10.30534/ijeter/2020/23822020.

18. Altoum AEA, Babker AMa, Osman AL, Ismail M, Lyashenko V. New Approach for Analysis the Correlation of Some Oxidative Markers in Type 2 Diabetes Mellitus by Data Wavelet Analysis. Indian Journal of Public Health Research \& Development, 2019; 10(11):2449-2455. 10.5958/09765506.2019.03975.5.

19. Ronco C, Navalesi P, Vincent JL. Coronavirus epidemic: preparing for extracorporeal organ support in intensive care. The Lancet Respiratory Medicine, 2020; 8(3):240241. 10.1016/S2213-2600(20)30060-6.

20. Benavides EM. Robust predictive model for Carriers, Infections and Recoveries (CIR): predicting death rates for CoVid-19 in Spain. arXiv preprint arXiv:2003.13890, 2020.

21. Kumar $P$ and et al., Forecasting the dynamics of COVID-19 Pandemic in Top 15 countries in April 2020 through ARIMA Model with Machine Learning Approach. Med Rxiv, 2020. 